PROCEEDINGS OF THE

AMERICAN MATHEMATICAL SOCIETY

Volume 137, Number 1, January 2009, Pages 287-295

S 0002-9939(08)09499-9

Article electronically published on June 30, 2008

\title{
ASYMMETRY OF CONVEX SETS WITH ISOLATED EXTREME POINTS
}

\author{
GABOR TOTH
}

(Communicated by Alexander N. Dranishnikov)

\begin{abstract}
When measuring asymmetry of convex sets $\mathcal{L} \subset \mathbf{R}^{n}$ in terms of inscribed simplices, the interior of $\mathcal{L}$ naturally splits into regular and singular sets. Based on examples, it may be conjectured that the singular set is empty iff $\mathcal{L}$ is a simplex. In this paper we prove this conjecture with the additional assumption that $\mathcal{L}$ has at least $n$ isolated extreme points on its boundary.
\end{abstract}

\section{Introduction AND STATEMENT OF RESUlts}

Throughout, we use standard notation and basic concepts in the theory of convex sets and functions 1, 5. Let $\mathcal{E}$ be a Euclidean vector space of dimension $n$. (We usually take $\mathcal{E}=\mathbf{R}^{n}$.) If $\mathcal{K} \subset \mathcal{E}$, then $\langle\mathcal{K}\rangle$ and $[\mathcal{K}]$ denote the affine span and convex hull of $\mathcal{K}$, respectively. For $\mathcal{K}=\left\{B_{0}, \ldots, B_{m}\right\}$ finite, $[\mathcal{K}]$ is a convex polytope. This polytope is an $m$-simplex if $B_{0}, \ldots, B_{m}$ are affinely independent, or equivalently, if $\operatorname{dim}[\mathcal{K}]=\operatorname{dim}\langle\mathcal{K}\rangle=m$. A convex set $\mathcal{L} \subset \mathcal{E}$ is a convex body if it has nonempty interior. Every convex set is a convex body in its affine span.

Let $\mathcal{L}$ be a compact convex body in $\mathbf{R}^{n}$ and $O$ a point in the interior of $\mathcal{L}$. As in [6. 7], we define a sequence of (affine) invariants $\left\{\sigma_{m}(\mathcal{L}, O)\right\}_{m \geq 1}$. Intuitively, $\sigma_{m}(\mathcal{L}, O)$ measures how lopsided $\mathcal{L}$ is in dimension $m$ viewed from $O$. Since $\mathcal{L}$ is compact and convex, given $C \in \partial \mathcal{L}$, we have $\langle O, C\rangle \cap \partial \mathcal{L}=\left\{C, C^{o}\right\}$, where $C^{o}$ is called the opposite of $C$ (with respect to $O$ ). We define the distortion function $\Lambda_{\mathcal{L}}=\Lambda: \partial \mathcal{L} \times \operatorname{int} \mathcal{L} \rightarrow \mathbf{R}$ by

$$
\Lambda(C, O)=\frac{d(C, O)}{d\left(C^{o}, O\right)}, \quad C \in \partial \mathcal{L}, O \in \operatorname{int} \mathcal{L},
$$

where $d$ is the Euclidean distance in $\mathbf{R}^{n}$. The distortion $\Lambda$ is a continuous function [6, 7]. By definition, $\Lambda\left(C^{o}, O\right)=1 / \Lambda(C, O)$.

The minimum distortion $\lambda(O)=\inf _{C \in \partial \mathcal{L}} \Lambda(C, O)$, as a function on the interior of $\mathcal{L}$, has been studied by many authors. (See Grünbaum [2] and the extensive references therein.) In particular, there are many lower estimates on Minkowski's measure of symmetry $\sup _{O \in \operatorname{int} \mathcal{L}} \lambda(O)$ and the derived measures $\lambda\left(O_{0}\right)$, where $O_{0}$ is the centroid, the centers of circumscribed and inscribed ellipsoids, the centroid of the surface area of $\partial \mathcal{L}$, and the curvature centroid.

Let $m \geq 1$. A finite multi-set $\left\{C_{0}, \ldots, C_{m}\right\}$ is called an $m$-configuration with respect to $O$ if $\left\{C_{0}, \ldots, C_{m}\right\} \subset \partial \mathcal{L}$ and $O \in\left[C_{0}, \ldots, C_{m}\right]$. The set of $m$-configurations

Received by the editors July 2, 2007, and, in revised form, January 2, 2008.

2000 Mathematics Subject Classification. Primary 52A05; Secondary 52A38, 52B11.

(C) 2008 American Mathematical Society 
is denoted by $\mathcal{C}_{m}(\mathcal{L}, O)$. We define

$$
\sigma_{m}(\mathcal{L}, O)=\inf _{\left\{C_{0}, \ldots, C_{m}\right\} \in \mathcal{C}_{m}(\mathcal{L}, O)} \sum_{i=0}^{m} \frac{1}{1+\Lambda\left(C_{i}, O\right)} .
$$

Since a 1-configuration is an opposite pair of points, we have $\sigma_{1}(\mathcal{L}, O)=1$.

An $m$-configuration $\left\{C_{0}, \ldots, C_{m}\right\}$ for which the infimum is attained is called minimal. Compactness implies that minimal configurations exist. $\sigma_{m}(\mathcal{L},):. \operatorname{int} \mathcal{L} \rightarrow \mathbf{R}$ is a continuous function ([7, Theorem $\mathrm{D})$. In what follows, we suppress $O$ when no confusion arises. In addition, we also suppress the dimension $n$; in particular, we write $\sigma(\mathcal{L})$ for $\sigma_{n}(\mathcal{L})$, etc.

In general, we obviously have

$$
\sigma_{m+k}(\mathcal{L}) \leq \sigma_{m}(\mathcal{L})+\frac{k}{1+\max _{\partial \mathcal{L}} \Lambda}, \quad m, k \geq 1 .
$$

For $m=n$, a configuration in $\mathcal{C}_{n+k}(\mathcal{L}, O), k \geq 1$, always contains a subconfiguration in $\mathcal{C}(\mathcal{L}, O)$ so that equality holds in (1.1). (See [8] for details.) In other words, the sequence $\left\{\sigma_{m}(\mathcal{L})\right\}_{m \geq 1}$ is arithmetic (with difference $1 /\left(1+\max _{\partial \mathcal{L}} \Lambda\right)$ ) from the $n$-th term onwards.

By [7] (Theorem B), for $m \geq 1$, we have

$$
1 \leq \sigma_{m}(\mathcal{L}) \leq \frac{m+1}{2}
$$

The lower bound $\sigma_{m}(\mathcal{L})=1$ is realized iff there exists an affine subspace $\mathcal{F} \subset \mathbf{R}^{n}$, $O \in \mathcal{F}$, of dimension $m$ such that $\mathcal{L} \cap \mathcal{F}$ is an $m$-simplex. For $m \geq 2$, the upper bound $\sigma_{m}(\mathcal{L})=(m+1) / 2$ is realized iff $\mathcal{L}$ is symmetric (with respect to $O$ ).

Thus, up to scaling, $\sigma_{m}(\mathcal{L}, O), m \geq 1$, are measures of symmetry in the sense of Grünbaum 2] since $\sigma_{m}$ is clearly continuous on the space of compact convex bodies with specified interior points and is also invariant under similarity transformations.

For estimates on the related symmetries of measure

$$
\inf _{\left\{C_{0}, \ldots, C_{m}\right\} \in \mathcal{C}_{m}(\mathcal{L}, O)} \sum_{i=0}^{m} \Lambda\left(C_{i}, O\right) \text { and } \inf _{\left\{C_{0}, \ldots, C_{m}\right\} \in \mathcal{C}_{m}(\mathcal{L}, O)} \prod_{i=0}^{m} \Lambda\left(C_{i}, O\right)
$$

(at least for $m=n$ ) see also Grünbaum [2].

We define the regular set $\mathcal{R} \subset \operatorname{int} \mathcal{L}$ as

$$
\mathcal{R}=\left\{O \in \operatorname{int} \mathcal{L} \mid \sigma(\mathcal{L}, O)<\sigma_{n-1}(\mathcal{L}, O)+\frac{1}{1+\max _{\partial \mathcal{L}} \Lambda(., O)}\right\} .
$$

An element of $\mathcal{R}$ is called a regular point. An interior point is called singular if it is not regular. By continuity of the functions in the defining inequality of $\mathcal{R}$, the set $\mathcal{R}$ is open in int $\mathcal{L}$ (and hence in $\mathbf{R}^{n}$ ). The structure of a compact convex body viewed from a regular point is technically much easier to deal with than when viewed from a singular point. For example, as shown below (Lemma 2.1), if $O$ is regular, then there exists a minimal $n$-configuration consisting of extreme points only. (Recall that a point on the boundary of $\mathcal{L}$ is called an extreme point if it is not contained in the interior of a boundary line segment.) This, for $\mathcal{L}$ a convex polytope, reduces the determination of $\sigma(\mathcal{L}, O)$ to a finite enumeration on the vertices of $\mathcal{L}$. Moreover, according to a result in [7], the distortion function $\Lambda(\mathcal{L},$.$) is concave$ on $\mathcal{R}$. Irrespective of regularity, concavity of the distortion function holds in 2dimensions [7. By contrast, there exists a 4-dimensional cone in which, due to the 
existence of singular points near the base, the distortion function is not concave [6]. It is therefore important to analyze when and where singularity does occur.

It is easy to show that $O \in \mathcal{R}$ iff the convex hull of every minimal configuration is an $n$-simplex with $O$ in its interior. (See 8 for details.) In addition, $\Lambda(., O)$ attains its local maximum at each configuration point.

Because of this, we will need the behavior of the boundary of $\mathcal{L}$ at a local maximum $C$ of $\Lambda(., O)$ as described in [7] (Section 7). For the moment we only use the fact that if $C$ is a smooth point of $\partial \mathcal{L}$ at which $\Lambda(., O)$ assumes a local maximum, then $C^{o}$ is also smooth and the tangent spaces at $C$ and $C^{o}$ to $\partial \mathcal{L}$ are parallel. If $C$ is not a smooth point, it is still true that there exist parallel supporting hyperplanes at $C$ and $C^{o}$. In particular, $\left[C, C^{o}\right]$ is an affine diameter in the sense of Grünbaum [2]. Thus, if $O$ is a regular point $O$ belongs to at least $n+1$ (affinely independent) affine diameters. To determine points with this property is an unsolved problem; in particular, it is not known whether or not the centroid has this property. For further results, see Grünbaum [2] and Kosiński [3, 4].

We denote by $\mathcal{L}_{0}$ the set of extreme points of $\mathcal{L}$. By a theorem of Minkowski, we have $\mathcal{L}=\left[\mathcal{L}_{0}\right]$. (See Theorem $\mathrm{D}$ in [5], p. 84.) We call an extreme point $C \in \mathcal{L}_{0}$ isolated if $C$ is not a limit point of $\mathcal{L}_{0}$. The following simple example is the motivation for our study:

Example. Let $\mathcal{L}$ have an isolated extreme point $C$, and assume that, away from $C, \partial \mathcal{L}$ is smooth. We claim that there are singular points in the interior of $\mathcal{L}$.

First, since $C$ is an isolated extreme point, the set of supporting hyperplanes $\mathcal{H}$ at $C$ such that $\mathcal{L} \cap \mathcal{H}=\{C\}$ is a nonempty open set (in the respective Grassmann manifold). This follows from the conical structure of $\mathcal{L}$ near $C$. (In fact, $\mathcal{L}$ is the convex hull of $\left[\mathcal{L}_{0} \backslash\{C\}\right]$ and the single point $C$; see Lemma 2.2 below.) For each $\mathcal{H}$ in this set, we consider the set of points $B \in \partial \mathcal{L}, B \neq C$, such that the tangent space of $\partial \mathcal{L}$ at $B$ is parallel to $\mathcal{H}$. Since $\mathcal{L}$ is convex and, away from $C$, its boundary is smooth, the union $\mathcal{B}$ of these sets is open in $\partial \mathcal{L}$. (In fact, $\partial \mathcal{L} \backslash \mathcal{B}$ is closed, as follows again from the conical structure of $\mathcal{L}$ near $C$.) The convex hull $[\mathcal{B}]$ intersects the interior of $\mathcal{L}$. Any point $O$ in this interior must be singular. Indeed, if $O$ were regular, then at least one point in a minimal configuration $\left\{C_{0}, \ldots, C_{n}\right\} \in \mathcal{C}(\mathcal{L}, O)$ would be contained in $\mathcal{B}$. This is a contradiction, since the tangent space at that point has no parallel translate tangent to $\partial \mathcal{L}$ at another smooth point.

The situation in the nonsmooth case is much more complicated. Our first result asserts that regularity of the interior of $\mathcal{L}$ along with the existence of isolated extreme points impose severe restrictions to the structure of $\mathcal{L}$.

Theorem 1.1. Let $\mathcal{L} \subset \mathbf{R}^{n}$ be a compact convex body with all interior points regular. Assume that $\mathcal{L}$ has (at least) two isolated extreme points $C_{0}$ and $C_{1}$. Then, for any plane $\tau$ that contains $C_{0}$ and $C_{1}$, the intersection $\mathcal{L} \cap \tau$ is either $\left[C_{0}, C_{1}\right]$ or a triangle with $\left[C_{0}, C_{1}\right]$ as a side.

An illustrative example to Theorem 1.1 to be discussed below is the following:

Example. Let $S \subset \mathbf{R}^{3}$ be the unit circle of the coordinate plane spanned by the first and second coordinate axes, and let $C_{ \pm}=(1,0, \pm 1)$. Let $\mathcal{L}$ be the convex hull $\left[S, C_{+}, C_{-}\right]$. Then $\mathcal{L}_{0}=(S \backslash\{(1,0,0)\}) \cup\left\{C_{ \pm}\right\}$. Clearly, $\mathcal{L}_{0}$ is not closed. Due to triangular intersections, we have $\sigma_{2}(\mathcal{L},)=$.1 . Hence [7, $\sigma(\mathcal{L},$.$) is concave on the$ whole interior of $\mathcal{L}$. 
Theorem 1.2. Let $\mathcal{L} \subset \mathbf{R}^{n}$ be as in Theorem 1.1. Assume that $\mathcal{L}$ has at least $n$ isolated extreme points. Then $\mathcal{L}$ is a simplex.

For $\mathcal{L}$ a convex polytope, the extreme points are the vertices and they are all isolated. Theorem 1.2 gives the following:

Theorem 1.3. Let $\mathcal{L} \subset \mathbf{R}^{n}$ be a convex polytope which is not a simplex. Then there are singular points in the interior of $\mathcal{L}$.

The proof will actually show that, if nonempty, the set of singular points has nonempty interior and its closure contains part of the boundary of $\mathcal{L}$.

\section{Proofs}

Let $\mathcal{L} \subset \mathbf{R}^{n}$ be a compact convex body. We first recall the notion of $k$-flat points on $\partial \mathcal{L}$ [7]. Let $C \in \partial \mathcal{L}$. We call an affine subspace $\mathcal{A} \subset \mathbf{R}^{n}$ a supporting flat at $C$ if $C \in \mathcal{A}$ and $\mathcal{A}$ is contained in a supporting hyperplane of $\mathcal{L}$ at $C$. Consider the set of supporting flats $\mathcal{A}$ at $C$ such that $\partial \mathcal{L} \cap \mathcal{A}$ is a compact convex body in $\mathcal{A}$ and $C$ is contained in its relative interior. Since $\mathcal{L}$ is convex, this set has a unique maximal element denoted by $\mathcal{A}_{C}$. We call $C$ a $k$-(dimensional) flat point if $\operatorname{dim} \mathcal{A}_{C}=k$. Clearly, $C$ is an extreme point iff $k=0$.

Lemma 2.1. Let $\mathcal{L} \subset \mathbf{R}^{n}$ be a compact convex body. If $O$ is a regular point of $\mathcal{L}$, then there exists a minimal configuration $\left\{C_{0}, \ldots, C_{n}\right\} \in \mathcal{C}(\mathcal{L}, O)$ consisting of extreme points.

Proof. Let $\left\{C_{0}, \ldots, C_{n}\right\} \in \mathcal{C}_{n}(\mathcal{L}, O)$ be minimal. Since $O$ is a regular point, $\left[C_{0}, \ldots, C_{n}\right]$ is an $n$-simplex containing $O$ in its interior and $\Lambda(., O)$ attains a relative maximum at each $C_{i}, i=0, \ldots, n$. Suppressing the index for simplicity, assume that a configuration point $C$ is not extremal. Then $C$ is a $k$-flat point for some $k>0, k=\operatorname{dim} \mathcal{A}_{C}$. Since $\Lambda(., O)$ attains a relative maximum at $C$, according to a result of [7] (the proposition in Section 7), the antipodal point $C^{o}$ is $l$-flat, $l \geq k$, and $\mathcal{A}_{C}$ is parallel to $\mathcal{A}_{C^{o}}$ in the sense that a translate of $\mathcal{A}_{C}$ is contained in $\mathcal{A}_{C^{o}}$.

Choose a point $C^{\prime}$ on the boundary of the compact convex body $\partial \mathcal{L} \cap \mathcal{A}_{C}$ in $\mathcal{A}$. Clearly, $C^{\prime}$ is a lower dimensional flat point than $C$. Since $\mathcal{A}_{C}$ is parallel to $\mathcal{A}_{C^{o}}, \Lambda(., O)$ is constant on $\left[C, C^{\prime}\right]$. Moving $C$ toward $C^{\prime}$ and replacing $C$ with the moved point, the configuration condition $O \in\left[C_{0}, \ldots, C_{n}\right]$ stays intact since $O$ is a regular point. Thus, replacing $C$ by $C^{\prime}$ in the configuration, we arrive at a minimal configuration with $C^{\prime}$ being a lower dimensional flat point than $C$. Proceeding inductively, we can replace each nonextremal point of the configuration with and extremal point without altering minimality. Lemma 2.1 follows.

Corollary. Let $\mathcal{L} \subset \mathbf{R}^{n}$ be a convex polytope and denote by $\mathcal{V}$ the set of vertices. Assume that $O$ is a regular point of $\mathcal{L}$. Then, we have

$$
\sigma(\mathcal{L}, O)=\min _{\left\{V_{0}, \ldots, V_{n}\right\} \in \mathcal{V}} \sum_{i=0}^{n} \frac{1}{1+\Lambda\left(V_{i}, O\right)} .
$$

Returning to the general setting, as in Section 1 , we let $\mathcal{L}_{0} \subset \partial \mathcal{L}$ denote the set of extreme points. We have $\mathcal{L}=\left[\mathcal{L}_{0}\right]$. Recall that an extreme point $C$ is isolated if $C$ has an open neighborhood disjoint from $\mathcal{L}_{0} \backslash\{C\}$. Our first task is to describe $\mathcal{L}$ near an isolated extreme point. 
Lemma 2.2. Let $\mathcal{L} \subset \mathbf{R}^{n}$ be a compact convex body and $\mathcal{L}_{0}$ the set of extreme points. Let $C \in \mathcal{L}_{0}$ be an isolated extremal point. Then

$$
U_{C}=\mathcal{L} \backslash \overline{\left[\mathcal{L}_{0} \backslash\{C\}\right]}
$$

is a relatively open set in $\mathcal{L}$ that contains $C$. For any $C^{\prime} \in U_{C} \cap \partial \mathcal{L}, C^{\prime} \neq C$, the line segment $\left[C, C^{\prime}\right]$ is on the boundary of $\mathcal{L}$, and it extends to a boundary line segment $\left[C, C^{\prime \prime}\right]$ with $C^{\prime \prime} \in\left[\mathcal{L}_{0} \backslash\{C\}\right]$.

Proof. Let $C$ be an isolated extreme point. For the first statement we need to show that

$$
C \notin \overline{\left[\mathcal{L}_{0} \backslash\{C\}\right]} .
$$

Assuming the contrary, we can select a sequence $\left\{C_{k}\right\}_{k \geq 1} \subset\left[\mathcal{L}_{0} \backslash\{C\}\right]$ converging to $C$. For each $k \geq 1$, we can write $C_{k}$ as a convex linear combination $\sum_{i=0}^{n} \lambda_{i k} C_{i k}$, where $C_{i k} \in \mathcal{L}_{0}, C_{i k} \neq C$. By compactness, we may assume that, for each $0 \leq i \leq n$, $C_{i k} \rightarrow C_{i}$ and $\lambda_{i k} \rightarrow \lambda_{i}$ as $k \rightarrow \infty$. Taking the limit, we obtain $C=\sum_{i=0}^{n} \lambda_{i} C_{i}$. Since $C$ is an extreme point, the only way this is possible is that this sum reduces to a single term. We obtain that $C_{i}=C$ for a specific $0 \leq i \leq n$, and so $C_{i k} \rightarrow C_{i}=C$ as $k \rightarrow \infty$. Hence $C$ is not isolated. (2.2) follows.

For the second statement, let $C^{\prime} \notin \overline{\left[\mathcal{L}_{0} \backslash\{C\}\right]}$ be a boundary point of $\mathcal{L}$. Since $\left[\mathcal{L}_{0}\right]=\mathcal{L}$, we can certainly write $C^{\prime}$ as a convex linear combination of $C$ and (finitely many) points in $\mathcal{L}_{0} \backslash\{C\}$. The point $C$ must participate in this linear combination with positive coefficient. Hence $C^{\prime}$ is in the interior of a segment $\left[C, C^{\prime \prime}\right]$, where $C^{\prime \prime} \in\left[\mathcal{L}_{0} \backslash\{C\}\right]$. Finally, since $C$ and $C^{\prime}$ are both boundary points of $\mathcal{L}$, the entire line segment $\left[C, C^{\prime \prime}\right]$ is on the boundary of $\mathcal{L}$. Lemma 2.2 follows.

Remark. Consider the second example above. Removing $C_{-}$from $\mathcal{L}_{0}$, we see that $\left[\mathcal{L}_{0} \backslash\left\{C_{-}\right\}\right]$is the positive cone $\left[S, C_{+}\right]$with the half-open segment $\left[(1,0,0), C_{+}\right)$ deleted. Its closure is $\overline{\left[\mathcal{L}_{0} \backslash\left\{C_{-}\right\}\right]}=\left[S, C_{+}\right]$, and hence $U_{C_{-}}=\left[S, C_{-}\right] \backslash[S]$. Notice that, for any $C^{\prime}$ in the interior of $\left[C_{-},(1,0,0)\right]$, the line segment $\left[C_{-}, C^{\prime}\right]$ extends beyond $\overline{U_{C_{-}}}$to $\left[C_{-}, C_{+}\right]$.

Lemma 2.3. Let $C$ be an isolated extreme point of $\mathcal{L}$ with associated open set $U_{C}$. Then, for every $O \in U_{C}$, there is a minimal configuration which contains $C$. In particular, if $O$ is regular, then $\Lambda(., O)$ takes a local maximum at $C$.

Proof. Let $O \in U_{C}$ and, as in Lemma 2.1, choose a minimal configuration consisting of extreme points. If $C$ does not participate in the configuration, then $O$ must be contained in $\left[\mathcal{L}_{0} \backslash\{C\}\right]$. This contradicts the assumption. Thus, $C$ is a point in the configuration. The last statement is clear.

For the next step we introduce some notation and recall some results in [7] (Section 7). Let $C$ be an isolated extreme point of $\mathcal{L}$. Let $\tau \subset \mathbf{R}^{n}$ be a plane passing through $C$ and an interior point $O \in U_{C}$ of $\mathcal{L}$. We consider the planar convex body $\mathcal{L} \cap \tau$ with isolated extreme point $C$. As Lemma 2.3 asserts, $\mathcal{L} \cap \tau$ contains an angular domain with vertex at $C$. We let $[C, P],[C, Q] \subset \partial \mathcal{L} \cap \tau$ denote the maximal side segments of this domain. We orient $\tau$ from $O$ such that the positive orientation corresponds to the sequence $P, C, Q$. As in Section 7 of [7, $\alpha=\alpha_{\tau}(C)$ is the angle $\angle O C Q$. In a similar vein, we let $\alpha^{o}=\alpha_{\tau}\left(C^{o}\right)$, where $\alpha^{o}$ is the angle with vertex at $C^{o}$ between the line segment $\left[C^{o}, O\right]$ and the right tangent at $C^{o}$ to the boundary of $\mathcal{L} \cap \tau$. 
From now on we assume that $U_{C}$ consists of regular points only. By Lemma 2.3, $\Lambda(., O)$ attains a local maximum at $C$, and so, by Corollary 1 of Section 7 in [7,

$$
\alpha \leq \alpha^{o} \text {. }
$$

For a boundary point $B$ of $\mathcal{L} \cap \tau$, let $0 \leq \phi(C) \leq \pi$ denote the angle between the left and right tangent lines at $B$ to $\partial \mathcal{L} \cap \tau$. Then we have $\phi(C)=\angle P C Q$. For $O$ close to a fixed interior point of $[C, P]$, the right tangent to $\mathcal{L} \cap \tau$ at $C^{o}$ intersects the extension of the line segment $[C, P]$ beyond $P$. We let $R$ denote this intersection point. From the triangle $\triangle C C^{\circ} R$, we obtain

$$
\phi(C)-\alpha+\alpha^{o}+\beta=\pi,
$$

where $\beta=\angle C^{o} R C$. Combining this with (2.3), we get

$$
\phi(C)+\beta \leq \pi .
$$

We now let $O$ approach a fixed interior point of $[C, P]$. We claim that $\beta$ approaches $\phi(P)$. In fact, as $O$ approaches a fixed interior point of $[C, P]$, the antipodal $C^{o}$ approaches $P$ along the boundary of $\mathcal{L} \cap \tau$, and the right tangent line at $C^{o}$ approaches the left tangent line at $P$. (See formula (6) in 5, p. 7.) We obtain the following:

Lemma 2.4. Let $\mathcal{L} \subset \mathbf{R}^{n}$ be a compact convex body, $C$ an isolated extreme point, and assume that $U_{C}$ consists of regular points. Then, for any plane passing through $C$ and an interior point of $\mathcal{L}$, we have

$$
\phi(C)+\phi(P) \leq \pi,
$$

where $\phi(C)$ and $\phi(P)$ are the tangential angles of $\mathcal{L} \cap \tau$ at $C$ and $P$, and $[C, P]$ is a maximal line segment on the boundary of $\mathcal{L} \cap \tau$.

In the lemma above, we call $P$ an adjacent point to the isolated extreme point $C$. $P$ is adjacent to $C$ if $[C, P]$ is a maximal line segment on the boundary of $\mathcal{L}$.

Proof of Theorem 1.1. We may assume that $\mathcal{L} \cap \tau$ is more than $\left[C_{0}, C_{1}\right]$, in which case $\mathcal{L} \cap \tau$ is a compact convex body with isolated extreme points $C_{0}$ and $C_{1}$. Let $P_{0}, Q_{0} \in \partial \mathcal{L} \cap \tau$ and $P_{1}, Q_{1} \in \partial \mathcal{L} \cap \tau$ be adjacent to $C_{0}$ and $C_{1}$, respectively. Orient $\tau$ and choose the labels such that (with respect to an(y) interior point of $\mathcal{L} \cap \tau$ ) $P_{0}, C_{0}, Q_{0}$ and $P_{1}, C_{1}, Q_{1}$ are positively oriented. Assume first that the adjacent points are all distinct, the right tangent at $Q_{0}$ and the left tangent at $P_{1}$ intersect at a point $X$, and the left tangent at $P_{0}$ and the right tangent at $Q_{1}$ intersect at a point $Y$. For the angle sum of the (convex) octagon $\left[P_{0}, C_{0}, Q_{0}, X, P_{1}, C_{1}, Q_{1}, Y\right]$ we have

$$
\phi\left(P_{0}\right)+\phi\left(C_{0}\right)+\phi\left(Q_{0}\right)+\beta+\phi\left(P_{1}\right)+\phi\left(C_{1}\right)+\phi\left(Q_{1}\right)+\gamma=6 \pi,
$$

where $\beta$ and $\gamma$ are the angles at $X$ and $Y$, respectively. On the other hand, by (2.4), we have

$$
\phi\left(C_{0}\right)+\phi\left(P_{0}\right), \phi\left(C_{0}\right)+\phi\left(Q_{0}\right), \phi\left(C_{1}\right)+\phi\left(P_{1}\right), \phi\left(C_{1}\right)+\phi\left(Q_{1}\right) \leq \pi .
$$

Adding these, we obtain

$$
2 \phi\left(C_{0}\right)+2 \phi\left(C_{1}\right)+\phi\left(P_{0}\right)+\phi\left(P_{1}\right)+\phi\left(Q_{0}\right)+\phi\left(Q_{1}\right) \leq 4 \pi .
$$

Comparing this with (2.5), we get

$$
\phi\left(C_{0}\right)+\phi\left(C_{1}\right)+2 \pi-\beta-\gamma \leq 0 .
$$


This is a contradiction. Notice that we get a contradiction even when $\beta=\pi$ or $\gamma=\pi$ (the cases when the corresponding tangents coincide), and even when $P_{0}=Q_{1}$ but $P_{1} \neq Q_{0}$, or when $P_{1}=Q_{0}$ but $P_{0} \neq Q_{1}$.

If $X$ or $Y$ do not exist, we can add additional supporting lines to boundary points of $\mathcal{L} \cap \tau$ and get a contradiction again.

Summarizing, we obtain $P_{0}=Q_{1}$ and $P_{1}=Q_{0}$. As a byproduct, we also obtain that $\mathcal{L} \cap \tau=\left[P_{0}, C_{0}, P_{1}, C_{1}\right]$. If $P_{0}, C_{0}, P_{1}, C_{1}$ are all distinct, then, by (2.4), $\left[P_{0}, C_{0}, P_{1}, C_{1}\right]$ is a parallelogram with $\left[C_{0}, C_{1}\right]$ as a diagonal. Finally, if these points are not distinct, then $\mathcal{L} \cap \tau$ is a triangle with $\left[C_{0}, C_{1}\right]$ as a side (and $P_{0}$ or $P_{1}$ is the other vertex).

It remains to show that the parallelogram intersection is impossible. As in Lemmas 2.1-2.3, we let $O \in U_{C_{0}}$ and consider a minimal configuration $\left\{C_{0}, C_{1}, \ldots, C_{n}\right\}$ $\in \mathcal{C}(\mathcal{L}, O)$ consisting of extreme points only. By the last statement of Lemma 2.2, $O$ is contained in the interior of the triangle $\left[P_{0}, C_{0}, P_{1}\right]$. Thus, the opposite $P_{0}^{o}$ is contained in $\left[C_{0}, P_{1}\right]$. Any point in the segment $\left[C_{0}, P_{0}^{o}\right]$ has the same distortion as $C_{0}$ since $\mathcal{L} \cap \tau$ is a parallelogram. Since $O$ and $\overline{\left[\mathcal{L}_{0} \backslash C_{0}\right]}$ are disjoint, there must be a point $C_{0}^{\prime} \in\left[C_{0}, P_{1}\right]$ for which $O$ is on the boundary of $\overline{\left[\left(\mathcal{L}_{0} \backslash\left\{C_{0}\right\}\right) \cup\left\{C_{0}^{\prime}\right\}\right]}$. Thus, $O$ is on the boundary of $\left[C_{0}^{\prime}, C_{1}, \ldots, C_{n}\right]$. Hence $\left\{C_{0}^{\prime}, \ldots, C_{n}\right\} \in \mathcal{C}(\mathcal{L}, O)$. It must be minimal with $C_{0}^{\prime} \in\left[C_{0}, P_{0}^{o}\right]$ since the distortion along $\left[P_{0}^{o}, P_{1}\right]$ increases. This, however, contradicts the regularity of $O$. Theorem 1.1 follows.

Proof of Theorem 1.2. Let $\mathcal{L} \subset \mathbf{R}^{n}$ be as in Theorem 1.1. For $1 \leq m<n$, let $\mathcal{P}_{m}$ be the following statement: If $C_{0}, \ldots, C_{m} \in \mathcal{L}$ are (distinct) isolated extreme points, then they are affinely independent, and, for any $(m+1)$-dimensional affine subspace $\tau \subset \mathbf{R}^{n}$ that contains $C_{0}, \ldots, C_{m}$, the intersection $\mathcal{L} \cap \tau$ is either $\left[C_{0}, \ldots, C_{m}\right]$ or an $(m+1)$-simplex with $\left[C_{0}, \ldots, C_{m}\right]$ as a side.

Notice that $\mathcal{P}_{1}$ is Theorem 1.1, and the second statement of $\mathcal{P}_{n-1}$ is Theorem 1.2. Therefore, Theorem 1.2 will follow by proving $\mathcal{P}_{m}$ by induction with respect to $m=1, \ldots, n-1$. Before the general induction step, it is convenient to have an intermediate step:

Lemma 2.5. Let $\mathcal{L}$ be as in Theorem 1.1. Assume that, for a fixed $2 \leq m<n, \mathcal{P}_{i}$, $1 \leq i<m$, hold. Let $C_{0}, \ldots, C_{m}$ be isolated extreme points of $\mathcal{L}$. Then, $C_{0}, \ldots, C_{m}$ are affinely independent and

$$
\mathcal{L} \cap\left\langle C_{0}, \ldots, C_{m}\right\rangle=\left[C_{0}, \ldots, C_{m}\right] .
$$

Proof. We first show affine independence. Since $\mathcal{P}_{m-1}$ holds, $C_{0}, \ldots, C_{m-1}$ are certainly affinely independent. Thus, the affine span $\tau=\left\langle C_{0}, \ldots, C_{m-1}\right\rangle \subset \mathbf{R}^{n}$ is $(m-1)$ dimensional. Applying $\mathcal{P}_{m-2}$ to $\tau$, we obtain that $\mathcal{L} \cap \tau$ is an $(m-1)$ simplex with $\left[C_{0}, \ldots, C_{m-2}\right]$ as a side. Since $C_{m-1} \notin\left\langle C_{0}, \ldots, C_{m-2}\right\rangle$ is an extreme point of $\mathcal{L}$, it is also an extreme point of $\mathcal{L} \cap \tau$. Thus, we have

$$
\mathcal{L} \cap \tau=\left[C_{0}, \ldots, C_{m-1}\right] .
$$

Now, $C_{m}$ cannot be in this set since it is an extreme point of $\mathcal{L}$ and thereby also an extreme point of $\mathcal{L} \cap \tau$. Thus, $C_{0}, \ldots, C_{m}$ are affinely independent. We now add $C_{m}$ to $\tau$ and set $\tau=\left\langle C_{0}, \ldots, C_{m}\right\rangle \subset \mathbf{R}^{n}$, an $m$ dimensional affine subspace. Applying $\mathcal{P}_{m-1}$, once again, $\mathcal{L} \cap \tau$ must be an $m$-simplex with $\left[C_{0}, \ldots, C_{m-1}\right]$ as a side. $C_{m}$ is an extreme point in $\mathcal{L}$ and also in $\mathcal{L} \cap \tau$. Equation (2.6) follows. 
We now return to the proof of the general induction step. Assume that, for a fixed $2 \leq m<n, \mathcal{P}_{i}, 1 \leq i<m$, hold. The first statement in $\mathcal{P}_{m}$ is contained in Lemma 2.5. To prove the second statement, let $\tau \subset \mathbf{R}^{n}$ be an $(m+1)$ dimensional affine subspace that contains $C_{0}, \ldots, C_{m}$. We may assume that $\mathcal{L} \cap \tau \neq\left[C_{0}, \ldots, C_{m}\right]$, since otherwise we are done. Equation $(2.6)$ and $\left[\mathcal{L}_{0}\right]=\mathcal{L}$ show that $\mathcal{L}$ contains an extreme point $C$ away from $\left\langle C_{0}, \ldots, C_{m}\right\rangle$. In other words, $C_{0}, \ldots, C_{m}, C$ are affinely independent, and the $(m+1)$-simplex $\left[C_{0}, \ldots, C_{m}, C\right]$ is contained in $\mathcal{L} \cap \tau$. It remains to show that

$$
\mathcal{L} \cap \tau=\left[C_{0}, \ldots, C_{m}, C\right]
$$

To do this, we will show that

$$
\left[C_{0}, \ldots, \widehat{C_{i}}, \ldots, C_{m}, C\right] \subset \partial \mathcal{L}, \quad 0 \leq i \leq m .
$$

First note that (2.8) implies (2.7). Indeed, (2.8) says that all the faces of $\left[C_{0}, \ldots\right.$, $\left.C_{m}, C\right]$ opposite to $C_{0}, \ldots, C_{m}$ are on the boundary of $\mathcal{L}$. If the face $\left[C_{0}, \ldots, C_{m}\right]$ were not on the boundary of $\mathcal{L}$, then there would be another extreme point of $\mathcal{L}$, say $C^{\prime} \in \partial \mathcal{L} \cap \tau$, on the side of $\left\langle C_{0}, \ldots, C_{m}\right\rangle \subset \tau$ opposite to $C$. By (2.8) with $C$ replaced by $C^{\prime}$, we would obtain that $\mathcal{L} \cap \tau=\left[C_{0}, \ldots, C_{m}, C, C^{\prime}\right]$ is a double simplex with common base $\left[C_{0}, \ldots, C_{m}\right]$. This clearly contradicts $\mathcal{P}_{1}$.

It remains to show (2.8). To do this, for $0 \leq i \leq m$, we let $\tau_{i}=\left\langle C_{0}, \ldots, \widehat{C_{i}}, \ldots\right.$, $\left.C_{m}, C\right\rangle \subset \tau$ and apply $\mathcal{P}_{m-1}$. Then (2.6) in Lemma 2.5 gives

$$
\mathcal{L} \cap \tau_{i}=\left[C_{0}, \ldots, \widehat{C_{i}}, \ldots, C_{m}, C\right], \quad 0 \leq i \leq m .
$$

In particular, for $0 \leq i<j \leq m$, the $(m-1)$-simplex

$$
\left[C_{0}, \ldots, \widehat{C_{i}}, \ldots, \widehat{C_{j}}, \ldots, C_{m}, C\right]
$$

is on the boundary of $\mathcal{L}$. Let $C^{\prime} \in \partial \mathcal{L}$ be a point in the interior of this $(m-1)$ simplex and consider the plane $\sigma=\left\langle C_{i}, C_{j}, C^{\prime}\right\rangle$. By $\mathcal{P}_{1}, \mathcal{L} \cap \sigma=\left[C_{i}, C_{j}, C^{\prime \prime}\right]$ for some $C^{\prime \prime} \in \partial \mathcal{L} \cap \sigma$ with $C^{\prime} \in\left[C_{i}, C_{j}, C^{\prime \prime}\right]$. We claim that $C^{\prime \prime}=C^{\prime}$. This will clearly imply (2.8).

First, $C^{\prime}$ cannot be in the interior of $\left[C_{i}, C_{j}, C^{\prime \prime}\right]$ since otherwise $C^{\prime}, C^{\prime \prime}$ and the unique intersection point $C^{\prime \prime \prime}=\left\langle C^{\prime}, C^{\prime \prime}\right\rangle \cap\left[C_{i}, C_{j}\right]$ would be three collinear points on $\partial \mathcal{L}$, so that, by convexity, $\left[C^{\prime \prime}, C^{\prime \prime \prime}\right]$ would be on the boundary of $\mathcal{L}$.

Thus, $C^{\prime}$ is on the boundary of $\left[C_{i}, C_{j}, C^{\prime \prime}\right]$, say $C^{\prime} \in\left[C_{i}, C^{\prime \prime}\right]$. On the other hand, $C^{\prime} \in\left[C_{0}, \ldots, \widehat{C_{i}}, \ldots, \widehat{C_{j}}, \ldots, C_{m}, C\right]$ and $C^{\prime \prime} \in\left[C_{0}, \ldots, \widehat{C_{j}}, \ldots, C_{m}, C\right]$ since $C_{i}, C^{\prime}, C^{\prime \prime}$ are collinear. As $\left[C_{0}, \ldots, \widehat{C_{i}}, \ldots, \widehat{C_{j}}, \ldots, C_{m}, C\right]$ is the side of the $m$ simplex $\left[C_{0}, \ldots, \widehat{C_{j}}, \ldots, C_{m}, C\right]$ opposite to $C_{i}, C^{\prime}=C^{\prime \prime}$ follows. The second statement of $\mathcal{P}_{m}$ and hence Theorem 1.2 follow.

\section{REFERENCES}

1. B. Grünbaum, Convex Polytopes, Springer, 2003. MR.1976856 (2004b:52001)

2. B. Grünbaum, Measures of symmetry for convex sets, Proc. Sympos. Pure Math., Vol. VII (1963) 233-270. MR0156259 (27:6187)

3. A. Koziński, On involution and families of compacta, Bull. Acad. Polon. Sci. Cl. III 5 (1954) 1055-1059. MR0103476 (21:2244)

4. A. Koziński, On a problem of Steinhaus, Fund. Math. 46 (1958) 47-59. MR0132539 (24:A2379)

5. A. Roberts and D. Varberg, Convex Functions, Academic Press, 1973. MR 0442824 (56:1201)

6. G. Toth, On the structure of convex sets with applications to the moduli of spherical minimal immersions, Contributions to Algebra and Geometry (to appear). 
7. G. Toth, On the shape of the moduli of spherical minimal immersions, Trans. Amer. Math. Soc. 358, No. 6 (2006) 2425-2446. MR2204039 (2007b:53128)

8. G. Toth, Simplicial intersections of a convex set and moduli for spherical minimal immersions, Michigan Math. J. 52 (2004) 341-359. MR2069804 (2005e:53097)

9. G. Toth, Finite Möbius Groups, Minimal Immersions of Spheres, and Moduli, Springer, 2002. MR.1863996(2002i:53082)

Department of Mathematics, Rutgers University, Camden, New Jersey 08102

E-mail address: gtoth@camden.rutgers.edu 